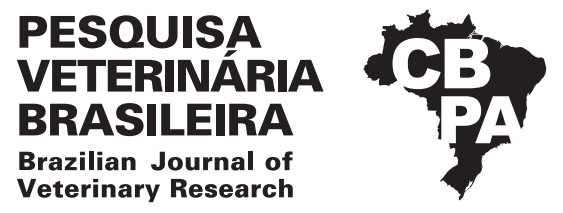

Pesq. Vet. Bras. 38(7):1405-1411, julho 2018 DOI: $10.1590 / 1678-5150-P V B-5534$

Artigo Original

ISSN 0100-736X (Print)

ISSN 1678-5150 (Online)

\title{
Neoplasmas cutâneos em cães: 656 casos (2007-2014) em Cuiabá, MT $^{1}$
}

\author{
Samara R. Lima2*, Mathias B. Stocco ${ }^{3}$, Leilane A.S. Rondelli ${ }^{3}$, Gustavo S. Silva ${ }^{2}$, \\ Roberto S. Lopes ${ }^{2}$, Fernando H. Furlan ${ }^{4}$, Edson M. Colodel ${ }^{4}$ e Caroline A. Pescador $^{4}$
}

ABSTRACT.- Lima S.R., Stocco M.B., Rondelli L.A.S., Silva G.S., Lopes R.S., Furlan F.H., Colodel E.M. \& Pescador C.A. 2018. [Cutaneous neoplasms in dogs: 656 cases (2007-2014) from Cuiabá, MT.] Neoplasmas cutâneas em cães: 656 casos (2007-2014) em Cuiabá, MT. Pesquisa Veterinária Brasileira 38(7):1405-1411. Laboratório de Patologia Veterinária, Faculdade de Agronomia, Medicina Veterinária e Zootecnia, Universidade Federal de Mato Grosso, Av. Fernando Corrêa da Costa 2367, Cuiabá, MT 78060-900, Brazil. E-mail: samara.rosolem.vet@gmail.com

Canine cutaneous neoplasms present high and relevant prevalence throughout Brazil. Here we determine the frequency and epidemiological characteristics of canine cutaneous neoplasms in dogs diagnosed at the Laboratory of Veterinary Pathology of the Federal University of Mato Grosso (LPV-UFMT), Cuiabá, from 2007 to 2014. The necropsy and biopsy protocols from canine cutaneous neoplasms diagnosed at the LPV-UFMT were reviewed. Data regarding age, sex, breed of affected dogs, anatomical location, size, histomorphological diagnosis and biological behavior of the tumors were evaluated through a descriptive statistical analysis. In addition, the $\chi 2$ test was used for associations between biological behavior and mass size. Of the 3566 examinations performed, 656 (18.4\%) were diagnosed as cutaneous tumors being adult and old dogs of Pit Bull, Boxer and Poodle races most affected. Among the diagnoses, 11 morphological patterns were the most prevalent, being mastocytoma, squamous cell carcinoma (SCC) and tumors of vascular origin most frequently reported. In relation to the anatomical site, the head was the most affected. Most of the benign tumors were less than $1 \mathrm{~cm}$ in diameter and the malignant were 3 to $5 \mathrm{~cm}$. The increase of neoplasms in adults and old age dogs may be associated to the sum of damage caused by carcinogenic agents and the immunological impairment.

INDEX TERMS: Neoplasms, dogs, tumours, dermatopathology, retrospective study, Cuiabá, pathology.

RESUMO.- Os neoplasmas cutâneos em cães apresentam elevada e relevante prevalência em todo o Brasil. Sendo assim, objetivou-se determinar a frequência e algumas características epidemiológicas dos neoplasmas cutâneos em cães diagnosticados no Laboratório de Patologia da

\footnotetext{
${ }^{1}$ Recebido em 21 de agosto de 2017.

Aceito para publicação em 30 de agosto de 2017.

${ }^{2}$ Programa de Pós-Graduação em Ciências Veterinárias, Faculdade de Veterinária, Universidade Federal de Mato Grosso (UFMT), Av. Fernando Corrêa da Costa 2367, Boa Esperança, Cuiabá, MT 78060-900, Brasil. *Autor para correspondência: samara.rosolem.vet@gmail.com

${ }^{3}$ Departamento de Medicina Veterinária Preventiva, Faculdade de Veterinária, EPILAB, Universidade Federal do Rio Grande do Sul, Av. Bento Gonçalves 9090, Porto Alegre, RS 91540-000, Brasil. E-mails: matiasnave@hotmail.com, leilane_go@hotmail.com

${ }^{4}$ Faculdade de Veterinária, Universidade Federal de Mato Grosso (UFMT), Av. Fernando Corrêa da Costa 2367, Boa Esperança, Cuiabá, MT 78060-900. E-mails: lopesdesouza.roberto@gmail.com, fhfurlan@gmail.com, moleta@ gmail.com, carolpescador@yahoo.com.br
}

Universidade Federal de Mato Grosso (LPV-UFMT), Cuiabá, entre os anos de 2007 a 2014. Foram revisados os protocolos de necropsias e biopsias do LPV-UFMT, de cães com neoplasmas cutâneos. Dados referentes à idade, sexo, raça dos cães afetados, localização anatômica, tamanho, diagnóstico histomorfológico e comportamento biológico foram coletados e analisados através de uma análise estatística descritiva. Adicionalmente o teste $\chi 2$, foi utilizado para associações entre comportamento biológico e tamanho da massa. Dos 3566 exames realizados, $656(18,4 \%)$ foram diagnosticados como tumores cutâneos sendo cães adultos e idosos das raças Pit Bull, Boxer e Poodle os mais acometidos. Dentre os 11 padrões morfológicos mais diagnosticados o mastocitoma, carcinoma de células escamosas (CCE) e os tumores de origem vascular foram os mais frequentemente relatados. Em relação ao sítio anatômico, a cabeça foi a mais acometida. A maioria dos tumores benignos apresentaram menos de $1 \mathrm{~cm}$ de diâmetro e os malignos de 3 a $5 \mathrm{~cm}$. 0 aumento de neoplasmas em adultos e idosos 
pode estar relacionado ao somatório de danos causados por agentes carcinogênicos e o comprometimento imunológico.

TERMOS DE INDEXAÇÃO: Neoplasmas cutâneas, tumores, caninos domésticos, dermatopatologia, estudo retrospectivo, patologia.

\section{INTRODUÇÃO}

Os distúrbios de pele representam aproximadamente $37,3 \%$ dos casos de diagnóstico histomorfológico em cães (Meirelles et al. 2010). Dentre as alterações, as neoplasias cutâneas são frequentemente relatados em vários estados brasileiros como Pará, Ceará, Paraíba, Minas Gerais, Paraná, Santa Catarina e Rio Grande do Sul assumindo prevalências que variam de $46,7 \%$ a 96,1\% (De Nardi et al. 2002, Bellei et al. 2006, Felisberto et al. 2010, Meirelles et al. 2010, Priebe et al. 2011, Andrade et al. 2012, Bastos et al. 2017), prevalecendo os de comportamento maligno (Meirelles et al. 2010, Fernandes et al. 2015).

A incidência de neoplasmas em pele de cães deve-se a alguns fatores como: (1) a pele ser o maior órgão do corpo e representar uma barreira física entre o ambiente e organismo (Fernandes et al. 2015), (2) estar diretamente exposta à fatores oncogênicos que, de alguma forma, promovem danos, propiciando o desenvolvimento de neoplasias (Martinez et al. 2006), (3) a pele apresentar elevado índice de degeneração celular e elevada mitose, favorecendo a ocorrência de mutações e propiciando o aparecimento de neoplasmas (Murphy 2006), (4) há facilidade na visualização da lesão, por parte dos tutores, quando comparado a outros órgãos (Merlo et al. 2008) e (5) devido à variedade dos componentes estruturais da epiderme e derme, nos quais cada tipo celular pode desenvolver um tipo de neoplasma (Bastos et al. 2017).

Acredita-se que fatores como a predisposição genética, histórico familiar, idade dos cães e a exposição à radiação ultravioleta favoreçam o aparecimento de neoplasias (Gallagher \& Lee 2006). Trabalhos internacionais demonstraram variação entre as prevalências dos tumores cutâneos caninos (Scott \& Paradis 1990, Goldschmidt \& Shofer 1992, Hill et al. 2006), provavelmente devido as diferenças geográficas associadas às diferenças na susceptibilidade, hábitos e a fatores ambientais (Souza 2005). Exemplos disso é o carcinoma de células escamosas (CCE), hemangioma e hemangiossarcoma, frequentemente mencionadas em cães que habitam áreas com elevada incidência solar (Gross et al. 2009).

Embora os neoplasmas cutâneas em cães representem uma importante fração diagnóstica de vários laboratórios de patologia animal (De Nardi et al. 2002, Bellei et al. 2006, Meirelles et al. 2010, Priebe et al. 2011, Andrade et al. 2012, Fernandes et al. 2015), há pouca utilização de técnicas especiais no diagnóstico, como por exemplo a imuno-histoquímica. Somado a isso, em muitos casos há falta de informações por parte dos tutores, dificultando a acurácia diagnóstica e estudos epidemiológicos (Bellei et al. 2006).

Embora o diagnóstico de neoplasmas cutâneos em cães seja rotineiro nos laboratórios de patologia veterinário, levantamentos epidemiológicos no Brasil são escassos, sendo observado apenas em 7 dos 26 estados, como previamente relatado. Sendo este, no conhecimento dos autores, o primeiro estudo epidemiológico dos tumores cutâneos em cães realizado no estado de Mato Grosso.

A identificação de risco para o desenvolvimento de câncer é um fator-chave na gestão da saúde do indivíduo e da população. Diante disso, este artigo visou determinar a frequência e algumas características epidemiológicas dos neoplasmas cutâneos diagnosticados histomorfologicamente em cães no LPV-UFMT, Cuiabá, no período de 2007 a 2014.

\section{MATERIAL E MÉTODOS}

Foi realizado um estudo retrospectivo de neoplasmas cutâneos em cães, entre os anos de 2007 a 2014, diagnosticados pelo Laboratório de Patologia Veterinária da Universidade Federal de Mato Grosso (LPV-UFMT), campus Cuiabá. Entre as informações encontradas nos arquivos, foram selecionados: raça, idade, sexo, tamanho da massa tumoral e localização anatômica da massa no corpo do animal e o diagnóstico.

Todos os casos eram provenientes de necropsias e biópsias, por incisão cirúrgica e/ou punch dermatológico, realizadas no Hospital Veterinário da UFMT (Hovet-UFMT) e clínicas veterinárias particulares das quatro mesorregiões do estado: Centro-sul $\left(15^{\circ} 35^{\prime} 56^{\prime \prime} \mathrm{S}\right.$ e $56^{\circ} 05^{\prime} 41^{\prime \prime} W ; 5^{\circ} 38^{\prime} 52^{\prime \prime}$ e $\left.56^{\circ} 07^{\prime} 59^{\prime \prime}\right)$; Sudoeste ( $14^{\circ} 37^{\prime} 40^{\prime \prime}$ e $\left.57^{\circ} 30^{\prime} 25^{\prime \prime}\right)$; Sudeste ( $16^{\circ} 28^{\prime} 16^{\prime \prime}$ e $54^{\circ} 38^{\prime} 13^{\prime \prime} \mathrm{W} ; 5^{\circ} 33^{\prime} 35^{\prime \prime}$ e e $\left.54^{\circ} 17^{\prime} 49^{\prime \prime} \mathrm{W}\right)$; e Norte $\left(11^{\circ} 52^{\prime} 22^{\prime \prime} \mathrm{S}\right.$ e $55^{\circ} 29^{\prime} 53^{\prime \prime} \mathrm{W}$; $13^{\circ} 50^{\prime} 18^{\prime \prime} \mathrm{S}$ e $56^{\circ} 04^{\prime} 50^{\prime \prime} \mathrm{W} ; 2^{\circ} 44^{\prime} 09^{\prime \prime} \mathrm{S}$ e $56^{\circ} 30^{\prime} 00^{\prime \prime} \mathrm{W}$ ) (IBGE 2017). Consideraram-se os diagnósticos morfológicos que constavam nos protocolos originais, buscando apenas quando necessário a padronização dos critérios escolhidos neste estudo através das classificações histomorfológicas descritas por Gross et al. (2009) e Goldschmidt \& Shofer (1992).

A localização das neoplasias foi anatomicamente separada em: cabeça, pescoço, membro, tórax, abdômen, períneo, cauda e escroto e múltiplas localizações, seguindo a metodologia de Fernandes et al. (2015). As faixas etárias foram divididas em: filhotes (até um ano de idade), adultos (de um a oito anos de idade) e idosos (acima de oito anos de idade) (Souza et al. 2006). 0 sexo foi descrito como macho ou fêmea.

Para a avaliação estatística foi realizada uma análise descritiva do banco de dados sendo posteriormente averiguada a distribuição de frequências e suas classificações. As associações entre comportamento biológico do tumor de pele e tamanho foi realizado através do teste qui-quadrado (Software R.3.1.1).

\section{RESULTADOS}

De 2007 a 2014 o LPV-UFMT diagnosticou 3.566 exames, originados de necropsia e biópsias, de cães e, destes, $656(18,4 \%)$ eram neoplasmas cutâneos. A prevalência destes tumores oscilou durante os oito anos estudados (Fig.1). Entre as 656 amostras, apenas $356(54,3 \%)$ havia registro da mesorregião originada, distribuindo-se em: 328 (50,0\%) da região Centro-Sul, 17 (2,6\%) Sudoeste, $6(0,9 \%)$ Sudeste e $5(0,8 \%)$ Norte (Fig.2).

A idade dos cães acometidos variou entre quatro meses e 18 anos (média de 7,7 anos), com predomínio $(50,2 \%)$ dos adultos. Fêmeas representaram 50,6\% dos cães e machos 49,4\% da população estudada. Não foi observada diferença significativa entre sexo, ocorrência de neoplasmas e idade (Quadro 1). No entanto, ao analisar o sexo em função das raças (Quadro 2), observou-se maior ocorrência em machos nas raças Shitzu (80\%) e Fila brasileiro $(64,7 \%)$ e fêmeas nas Cocker Spaniel (66,7\%), Rotweiller (61,3\%) e Poodle (61,1\%).

Os cães com raça definida (CRD) apresentaram-se em quantidade numérica superior aos sem raça definida (SRD) (463/656; [70,57\%]) e perfizeram 36 variações raciais. As 10 de maior ocorrência (54\%) estão listadas no quadro 2. 
Eercentagem

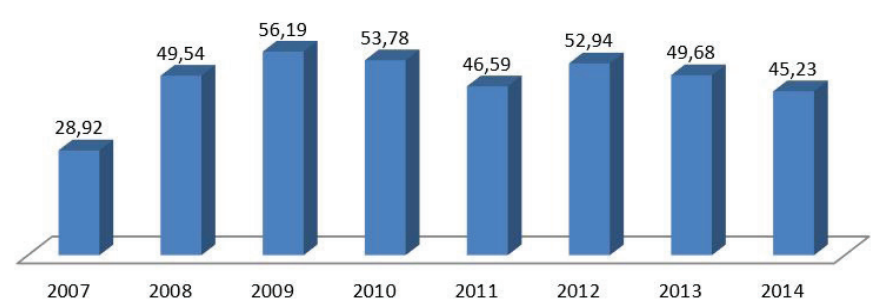

Fig.1. Prevalência dos 656 neoplasmas cutâneos de cães diagnosticados de 2007 a 2014 no Laboratório de Patologia Veterinária, Universidade Federal de Mato Grosso.

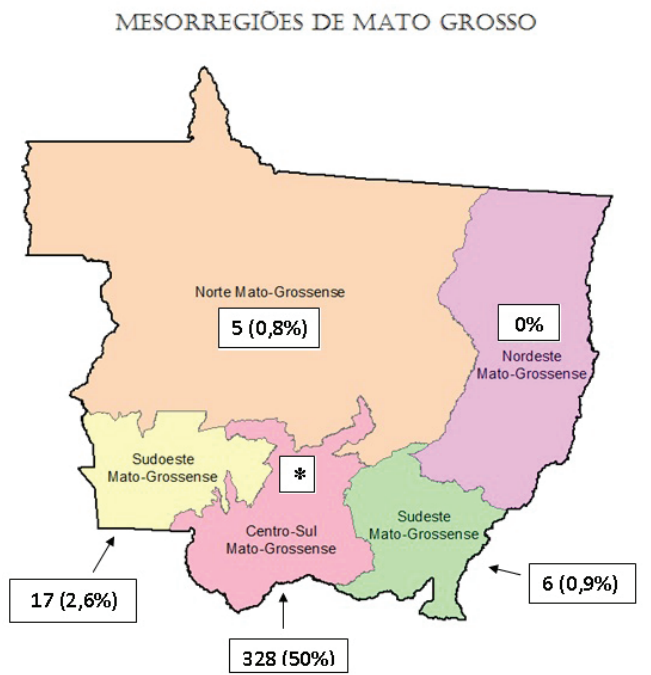

Fig.2. Origem dos 656 neoplasmas cutâneos de cães diagnosticados no Laboratório de Patologia Veterinária, Universidade Federal de Mato Grosso. Ni = Dado não informado; * Local aproximado da cidade de Cuiabá. Fonte: Adaptado do IBGE, 2017.

As 26 raças não inclusas, devido à baixa ocorrência, foram: Fox Paulistinha (1,37\%), Dog Alemão e Bassed Hound (1,21 cada), Maltês (0,91\%), Lhasa apso, York Shire, Schnauzer e Sharpei $(0,76 \%$ cada), Beagle e Dobermann ( $0,6 \%$ cada), Dálmata e Pug $(0,45 \%$ cada), Pastor Belga, Spitz Alemão, Golden Retriever, Scottish terrier e Bull terrier (0,3\% cada), American Staffordshire Terrier, Border collie, Blue hiller, Bulldog Alemão, Dog Argentino, Pointer, Weimarainer e West Highland $(0,15 \%$ cada).

No diagnóstico histomorfológico (Quadro 3) predominou os tumores mesenquimais (41\%) seguidos dos epiteliais, tumores de células redondas (TCR) e melanocíticos $(31,7 \%$, $21,5 \%$ e $5,8 \%$, respectivamente). Os mesenquimais mais diagnosticados foram: mastocitoma $(30,11)$, hemangioma $(20,82)$, hemangiossarcoma $(17,84 \%)$ e lipoma $(11,15 \%)$; os epiteliais: carcinoma de células escamosas (CCE) $(27,4 \%)$, tricoblastoma $(13,46 \%)$ e adenoma de glândula sebácea (9,61\%); TCR: linfoma (38,29\%), histiocitoma $(26,24 \%)$ e tumor venéreo transmissível (TVT) (13,47\%); e melanocíticos:
Quadro 1. Total de tumores da pele diagnosticados no Laboratório de Patologia Veterinária, Universidade Federal de Mato Grosso, distribuído por idade em relação ao sexo, de 2007 a 2014

\begin{tabular}{|c|c|c|c|c|c|}
\hline \multirow{3}{*}{ Idade cães } & \multicolumn{4}{|c|}{ Neoplasmas da pele } & \multirow{3}{*}{$\frac{\text { Total }}{\text { № }}$} \\
\hline & \multicolumn{2}{|c|}{ Fêmeas } & \multicolumn{2}{|c|}{ Machos } & \\
\hline & № & $\%$ & № & $\%$ & \\
\hline Até 1 ano (jovens) & $6(1,8)$ & 1,81 & $3(1,0)$ & 0,93 & 9 \\
\hline 1-8 anos (adultos) & $165(49,7)$ & 49,70 & $164(50,6)$ & 50,62 & 329 \\
\hline$>8$ anos (idosos) & $112(33,8)$ & 33,74 & $108(33,3)$ & 33,33 & 220 \\
\hline Não informado (NI) & $49(14,7)$ & 14,75 & $49(15,1)$ & 15,12 & 98 \\
\hline TOTAL & $332(100)$ & 100 & $324(100)$ & 100 & 656 \\
\hline
\end{tabular}

melanoma (92,1\%). Estes 11 diagnósticos representaram $70,9 \%$ dos 43 tipos tumorais diagnosticados neste estudo.

Ao relacionar o diagnóstico histomorfológico e sexo (quadro 3) observou-se que em machos havia maior ocorrência de adenoma e carcinoma da glândula hepatóide $(80 \%$ e $71,4 \%$ respectivamente), adenoma de glândula lacrimal (80\%), histiocitoma $(64,9 \%)$ e TVT $(63,2 \%)$; e em fêmeas o tricoepitelioma $(100 \%)$, lipoma $(73,3 \%)$, melanoma $(65,7 \%)$ e melanocitoma $(66,7 \%)$.

0 diagnóstico histomorfológico em função da raça (Quadro 3) apontou que os neoplasmas originados de estruturas do folículo piloso foram mais observados nas raças Poodle e Lhasa Apso, glandulares na Poodle, vasculares, linfoma e histiocitoma no Pit Bull, melanocíticos no Rottweiler e mastocitoma no Boxer.

Com relação às raças em função do diagnóstico histomorfológico (Quadro 2): o Fila Brasileiro, Boxer e Labrador Retriever foram as mais acometidas por mastocitoma, com 35,1\%,25\% e 16,6\%, respectivamente; o American Pit Bull Terrier (Pit Bull) por hemangiossarcoma (22,8\%), CCE e hemangioma (19,49\%); o Poodle por adenoma sebáceo $(20,3 \%)$ e tricoblastoma (12,9\%); e o Shitzu por tricoblastoma (40\%). A idade média, sexo e principais diagnósticos histomorfológico nas 10 raças de maior ocorrência estão detalhadas no quatro 2 .

Quanto ao comportamento biológico há predomino da forma benigna (380/656; 57,93\%). Do total de neoplasmas cutâneos inclusos, apenas $441(67,23 \%)$ apresentavam registro do tamanho da massa e, destes, 253/441 (57,4\%) eram benignos (quadro 4). Há diferença estatística na relação entre o tamanho dos tumores e sua classificação biológica ( $p=0.0008206)$. Um total de 59,6\% (112/188) dos neoplasmas malignos apresentavam de 3 a $5 \mathrm{~cm}$ de diâmetro, sugerindo malignidade.

Quanto à localização anatômica: 0 papiloma, melanoma e tricoblastoma acometeram predominantemente à cabeça (76,9\%, 52,9\% e 44,4\% respectivamente); o hemangiossarcoma, hemangioma, mastocitoma e CCE o abdômen $(31,7 \%$, $27,7 \%, 24,3 \%$ e $24 \%$ ); e o Adenoma e carcinoma de células hepatóides, TVT, hemangiossarcoma e hemangioma o períneo $(100 \%, 100 \%, 94,7 \%, 29,3 \%$ e 23,4\%) (Quadro 3). Entre os tumores mesenquimais houve predomínio de ocorrência nos membros (50/269) e abdômen (47/269), epiteliais a cabeça (65/208) e períneo (34/208), TCR múltiplos locais (42/141) e melanocítico a cabeça (16/38). 0 local de maior ocorrência entre os 656 neoplasmas cutâneos é a cabeça, seguida pelos membros, e o de menor é a cauda seguida pelo pescoço. 
Quadro 2. Descrição das 20 principais raças caninas em função da quantidade de animais, idade média, sexo e principais diagnósticos histomorfológicos de tumores da pele em cães diagnosticados de 2007 a 2014 no Laboratório de Patologia Veterinária, Universidade Federal de Mato Grosso

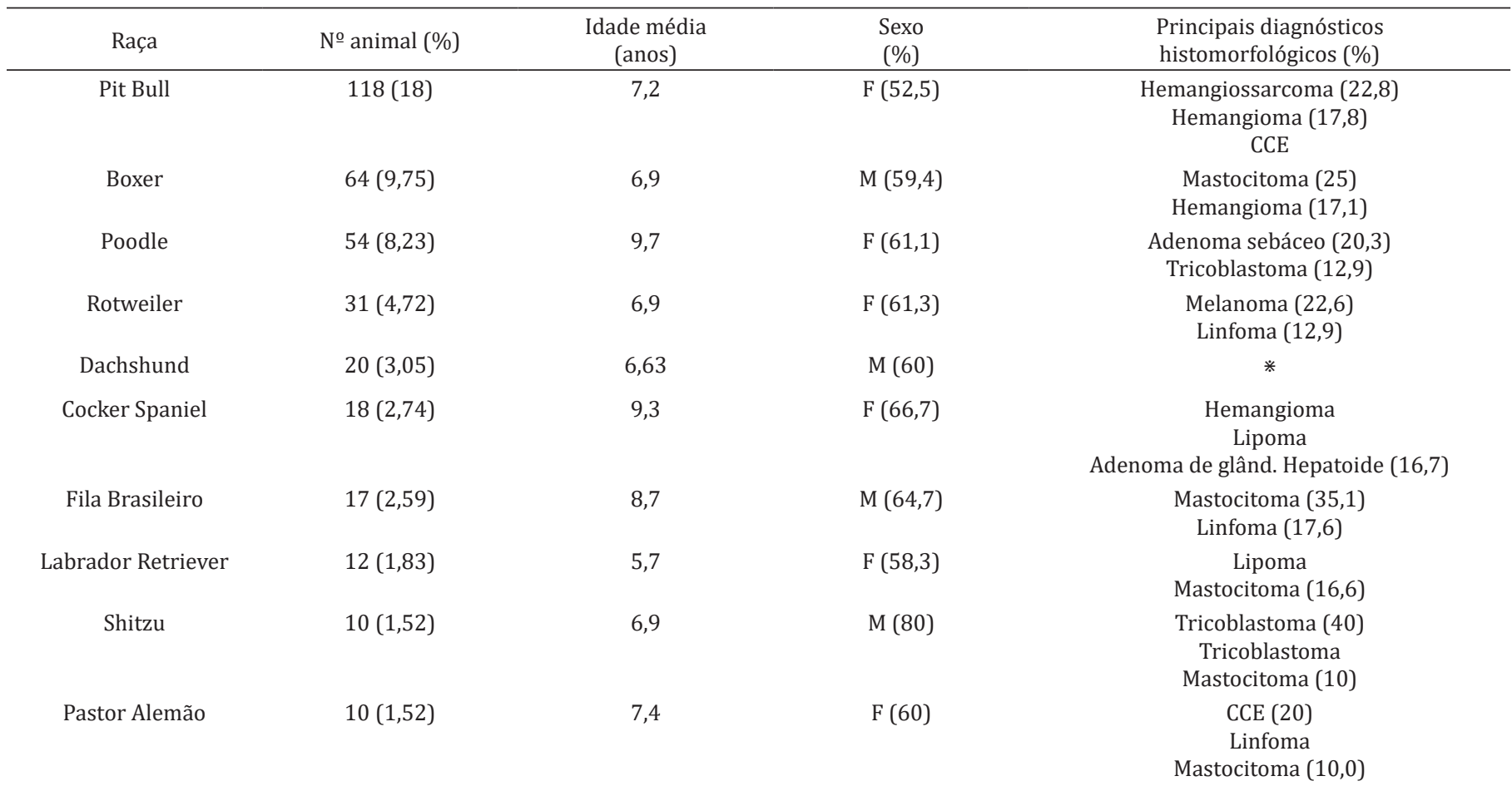

Pit Bull = American Pit Bull Terrier, CCE = carcinoma de células escamosas, * = há mais de três diagnósticos tumoral com a mesma porcentagem de ocorrência.

\section{DISCUSSÃO}

A incidência dos neoplasmas cutâneos em cães apresentou oscilação, no entanto é possível notar aumento na casuística, semelhante ao observado em Santa Maria (Souza et al. 2006) e Fortaleza (Bastos et al. 2017). Acredita-se que este aumento esteja relacionado à: crescente preocupação dos clínicos veterinários e tutores em fazer o diagnóstico das lesões de pele (Viana et al. 2014); e à aproximação entre cão e tutor, favorecendo a visualização precoce das massas (Bastos et al. 2017).

A mesorregião com maior casuística (50\%) foi à centro-sul (Cuiabá e Várzea Grande), provavelmente decorrente da proximidade geográfica ao LPV-UFMT, situado na cidade de Cuiabá. Os cães adultos ( 1 a 8 anos) foram os mais afetados $(50,15 \%)$ por neoplasma cutâneo, seguido pelos idosos (33,54\%), corroborando com Meirelles et al. (2010) e Bastos et al. (2017). Sugere-se que a prolongada exposição da pele à carcinógenos ambientais (Bellei et al. 2006) e o comprometimento do sistema imunológico (Modiano \& Breen 2007) são fatores envolvidos no aparecimento das neoplasias nesta faixa etária.

Não foi observada diferença estatística entre o diagnóstico e a predisposição sexual, no entanto os adenomas e carcinomas hepatóides foram mais observados em machos e os lipomas em fêmeas. Dados similares foram observados por Meirelles et al. (2010) e Hobert et al. (2013) respectivamente. No presente estudo não há dados em relação à castração dos animais, impossibilitando associar a neoplasia com a produção hormonal e a idade, como o realizado por Torres de la Riva et al. (2013).

Entre os cães CRD houve predomínio de Pit Bull, Boxer e Poodle, semelhante aos demais trabalhos realizados (Andrade et al. 2012, Fernandes et al. 2015). A elevada incidência de neoplasmas em cães CRD sugere predisposição genética (Gross et al. 2009), resultando em múltiplas gerações de cães acometidos pelo mesmo neoplasma (Davis \& Ostrander 2014). Como o observado neste estudo, o Boxer é um exemplo de raças que é comumente acometida por mastocitoma (Pizzoni et al. 2017) e hemangiossacoma (Meirelles et al. 2010); e o Pit Bull por tumores vasculares e CCE (Fernandes et al. 2015).

Adicionalmente, a presença de tumores vasculares na raça Pit Bull pode ser favorecida pela exposição da pele à radiação solar devida ao pelo curto e pouca pigmentação cutânea que estes animais possuem (Goldschmidt \& Shofer 1992). Entretanto, é importante salientar que não possuímos o número total de cães desta raça, o que nos impossibilita descartar a possibilidade de vício ambiental nas amostras.

Embora o TVT não tenha sido apontado como um dos cinco tumores mais diagnosticados, a sua frequência foi alta $(2,9 \%)$ quando comparada a estudos realizados em Porto Alegre e Santa Maria (Souza et al. 2006, Meirelles et al. 2010). Entretanto, considerada baixa quando comparada a Uberlândia (Fernandes et al. 2015), Paraíba (Andrade et al. 2012), Belém (Priebe et al. 2011), Curitiba (De Nardi et al. 2002) e Lages (Bellei et al. 2006). Por disseminar-se durante o coito (Komnenou et al. 2015), ou contato físico (Lima et al. 2013), este neoplasma apresenta-se com frequência elevada em áreas com grande quantidade de cães errantes. Acreditamos 
Quadro 3. Descrição dos tumores cutâneos de cães em função da quantidade de animais, raça mais acometida, local anatômico e sexo diagnosticados no Laboratório de Patologia Veterinária da Universidade Federal de Mato Grosso, entre 2007 e 2014

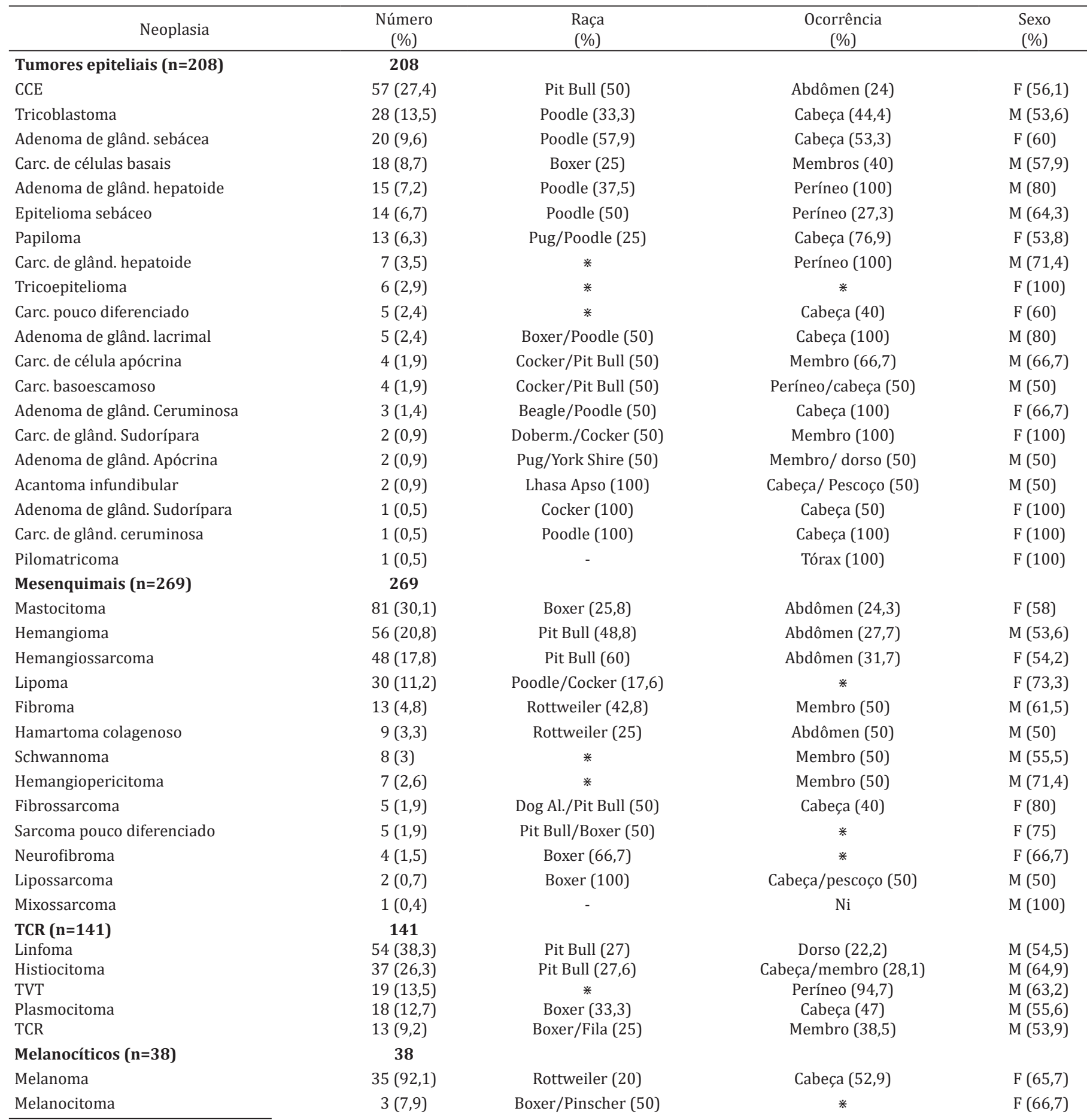

Carc. $=$ Carcinoma, CCE $=$ Carcinoma de Células Escamosas, TVT $=$ Tumor Venéreo Transmissível Canino, TCR $=$ Tumor de Células Redondas pouco diferenciadas, Gland. = Glândula, * = há pelo menos três especificações com a mesma ocorrência, Pit Bull = American Pit Bull Terrier, Fila = Fila Brasileiro, Pinscher $=$ Pinscher miniatura, Cocker $=$ Cocker Spaniel, Dog Al. $=$ Dog Alemão, $\mathrm{Ni}=$ não informado, Membro $=$ membros anteriores e posteriores, Acantoma infundibular = Acantoma infundibular queratinizante, Doberm. $=$ Dobermann .

que a incidência de TVT em Cuiabá está sendo subestimada, pois grande parte destes diagnósticos é decorrente da análise citomorfológica, as quais não foram incluídas neste estudo.

Neste trabalho, os melanomas representaram $5,3 \%$ (35/656) dos diagnósticos, enquanto os CCE perfizeram
$8,7 \%$, sendo semelhante ao levantamento de neoplasia em pele de cães realizado em Fortaleza (Bastos et al. 2017). 0 risco de desenvolver melanoma aumenta quando há exposição solar intensa de forma crônica ou ocasional (Armstrong \& Kricker 1993) ou queimaduras solares 
Quadro 4. Correlação entre comportamento biológico e tamanho tumoral de 441 de 656 tumores da pele em cães diagnosticados no Laboratório de Patologia Veterinária, Universidade Federal de Mato Grosso, de 2007 a 2014

\begin{tabular}{|c|c|c|c|c|c|}
\hline \multirow{2}{*}{$\begin{array}{l}\text { Tamanho } \\
\text { do tumor }\end{array}$} & \multicolumn{2}{|c|}{ Benigno } & \multicolumn{2}{|c|}{ Maligno } & \multirow{2}{*}{$\frac{\text { Total }}{\mathrm{N}}$} \\
\hline & $\mathrm{N}$ & $\%$ & $\mathrm{~N}$ & $\%$ & \\
\hline$<1 \mathrm{~cm}$ & 43 & 16,99 & 13 & 6,91 & 56 \\
\hline $1 \mathrm{a} 2 \mathrm{~cm}$ & 95 & 37,54 & 63 & 33,52 & 158 \\
\hline 3 a $5 \mathrm{~cm}$ & 69 & 27,28 & 81 & 43,08 & 150 \\
\hline$>5 \mathrm{~cm}$ & 46 & 18,19 & 31 & 16,49 & 77 \\
\hline Total & 253 & 100 & 188 & 100 & 441 \\
\hline
\end{tabular}

(Oliveria et al. 2006). Em humanos, há 25 vezes mais risco de desenvolver melanoma em pessoas brancas do que em negras (American Cancer Society 2015). Neste trabalho, o Rottweiler foi a raça mais acometida por melanoma, provavelmente por serem mantido nos quintas das casas devido ao grande porte sendo utilizados para a proteção.

Diferente de outros estudos (Fernandes et al. 2015, Bastos et al. 2017), neste trabalho a maioria dos tumores apresentaram comportamento benigno $(57,93 \%)$ e menos de $1 \mathrm{~cm}$ de tamanho (46,3\%). Dado similar foi observado por Sorenmo et al. (2009), no qual a maioria dos tumores benignos eram pequenos no momento do diagnóstico, reforçando o resultado do presente estudo no qual há predomínio de tumores benignos com menos de $1 \mathrm{~cm}$ de diâmetro e malignos de 3 a $5 \mathrm{~cm}$.

Devido à variedade de células epiteliais que compõem a pele (Fernandes et al. 2015), esperava-se que os neoplasmas de origem epitelial fossem os mais prevalentes. Entretanto, os mesenquimais foram os mais frequentemente diagnosticados, provavelmente devido ao mastocitoma, o qual sozinho perfez um total de $30,11 \%$ de todos os tumores diagnosticados. As diferenças entre as prevalências nestes grupos possivelmente é um reflexo da população canina e a influência ambiental (Mukaratirwa et al. 2005).

A variação na ocorrência tumoral nos diferentes sítios anatômicos sugere haver predisposição ao desenvolvimento neoplásico em determinados locais (Kaldrymidou et al. 2002). Os mesenquimais predominaram na região do tronco e pouca ocorrência na cabeça e pescoço. Acredita-se que isso ocorra devido os três principais diagnósticos deste grupo (mastocitoma, hemangioma e lipoma) estar localizados em menos de $10 \%$ nesta região (Kaldrymidou et al. 2002). 0 CCE predominante $(42,1 \% \%)$ em abdômen é comum em pele despigmentada, ou apenas parte dela, (Fernandes et al. 2015). É interessante ressaltar que o melanoma oral apresenta comportamento mais agressivo (Gross et al. 2009), enquanto o que ocorre em pele com pelo tendem a menor agressividade (Bergman 2007), evidenciando a importância da localização anatômica no comportamento e predisposição tumoral.

\section{CONCLUSÕES}

Tumores cutâneos em cães apresentam prevalência de 18,4\%. Houve predomínio de 11 padrões morfológicos distintos, sendo o mastocitoma, CCE e tumores vasculares os mais prevalentes.

Pit Bull e boxer foram as raças mais acometidas.

A maioria dos neoplasmas cutâneos de $1 \mathrm{~cm}$ de diâmetro é benigna e localiza-se na cabeça.
A idade, a raça e o sexo são fatores que influenciam o desenvolvimento de alguns neoplasmas cutâneos de cães.

Agradecimentos.- À Coordenação de Aperfeiçoamento de Pessoal de Nível Superior (CAPES), pelo auxílio financeiro durante a execução deste trabalho.

\section{REFERÊNCIAS}

American Cancer Society 2015. Cancer Facts and Figures. Disponível em <http://www.cancer.org> Acesso em 2 mar. 2017.

Andrade R.L.F.S., Oliveira D.M., Dantas A.F.M., Souza A.P., Nóbrega Neto P.I. \& Riet-Correa F. 2012. Tumores de cães e gatos diagnosticados no semiárido da Paraíba. Pesq. Vet. Bras. 32(10):1037-1040. <http://dx.doi.org/10.1590/ S0100-736X2012001000016>

Armstrong B.K. \& Kricker A. 1993. How much melanoma is caused by sun exposure? Melanoma Res. 3(6):395-401.<http://dx.doi.org/10.1097/00008390199311000-00002><PMid:8161879>

Bastos R.S.C., Farias K.M., Lopes C.E.B., Pacheco A.C.L. \& Viana D.A. 2017. Estudo retrospectivo de neoplasias cutâneas em cães da região metropolitana de Fortaleza. Revta Bras. Hig. San. Anim. 11(1):39-53. <http://dx.doi. org/10.5935/1981-2965.20170005>

Bellei M.H.M., Neves D.D., Gava A., Liz P.P. \& Pilati C. 2006. Prevalência de neoplasias cutâneas diagnosticadas em caninos no estado de Santa Catarina, Brasil, no período entre 1998 a 2002. Revta Ciênc. Agrovet. 5(1):73-79.

Bergman P.J. 2007. Canine oral melanoma. Clin. Tech. Small Anim. Pract. 22(2):5560. <http://dx.doi.org/10.1053/j.ctsap.2007.03.004><PMid:17591290>

Davis B.W. \& Ostrander E.A. 2014. Domestic dogs and cancer research: a breed-based genomics approach. ILAR J. 55(1):59-68. <http://dx.doi. org/10.1093/ilar/ilu017> <PMid:24936030>

De Nardi A.B., Rodaski S., Sousa R.S., Costa T.A., Macedo T.R., Rodigheri S.M., Rios A. \& Piekarz C.H. 2002. Prevalência de neoplasias e modalidades de tratamentos em cães, atendidos no hospital veterinário da universidade federal do Paraná. Arch. Vet. Sci. 7(2):15-26. <http://dx.doi.org/10.5380/ avs.v7i2.3977>

Felisberto A.C.T., Carvalho G.D., Favarato E.S. \& Silva A.S.A. 2010. Casuística de cães atendidos com suspeita de neoplasias no Hospital Veterinário da Univiçosa, no período de 2007 a 2009. Anais II Simpósio Prod. Acadêmica. Viçosa 2(1):177-182.

Fernandes C.C., Medeiros A.A., Magalhães G.M., Juan Szabó M.P., Queiroz R.P., Silva M.V.A. \& Soares N.P. 2015. Frequência de neoplasias cutâneas em cães atendidos no hospital veterinário da universidade federal de uberlândia durante os anos 2000 a 2010. Biosc. J. 31(2):541-548. <http://dx.doi. org/10.14393/BJ-v31n2a2015-22371>

Gallagher L.T.K. \& Lee T.K. 2006. Adverse effects of ultraviolet radiation: a brief review. Prog. Biophys. Mol. Biol. 92(1):119-131. <http://dx.doi. org/10.1016/j.pbiomolbio.2006.02.011><PMid:16580054>

Goldschmidt M.H. \& Shofer F.S. 1992. Skin Tumors of the Dog and Cat. 2nd ed. Pergamon, Oxford, p.45-117.

Gross T.L., Lhrke P.J., Walder E.J. \& Affolter V.K. 2009. Doenças de Pele do Cão e do Gato: diagnostico clinico e histopatológico. $2^{\text {a }}$ ed. Roca, São Paulo. 889p.

Hill P.B., Lo A., Eden C.A.N., Huntley S., Morey V., Ramsey S., Richardson C., Smith D.J., Sutton C., Taylor M.D., Thorpe E., Tidmarsh R. \& Williams V. 2006. Survey of the prevalence, diagnosis and treatment of dermatological conditions in small animals in general practice. Vet. Rec. 158(16):533-539. <http://dx.doi.org/10.1136/vr.158.16.533> <PMid:16632525>

Hobert M.K., Brauer C., Dziallas P., Gerhauser I., Algermissen D., Tipold A. \& Stein V.M. 2013. Infiltrative lipoma compressing the spinal cord in 2 Large-Breed dogs. Can. Vet. J. 54(1):74-78. <PMid:23814306>

IBGE 2017. Instituto Brasileiro de Geografia e Estatística. Mato Grosso. Disponível em <http://cidades.ibge.gov.br/xtras/perfil.php?codmun=510340> Acesso em 2 mar. 2017. 
Kaldrymidou H., Leontides L., Koutinas A.F., Saridomichelakis M.N. \& Karayannopoulou M. 2002. Prevalence, distribution and factors associated with the presence and the potential for malignancy of cutaneous neoplasms in 174 dogs admitted to a clinic in northern Greece. J. Vet. Med. 49(2):87-91. <http://dx.doi.org/10.1046/j.1439-0442.2002.jv408.x><PMid:11958472>

Komnenou A.T., Thomas A.L., Kyriazis A.P., Poutahidis T. \& Papazoglou L.G. 2015. Ocular manifestations of canine transmissible venereal tumour: a retrospective study of 25 cases in Greece. Vet. Rec. 176(20):523. <http:// dx.doi.org/10.1136/vr.102968><PMid:25888603>

Lima T.B., Marinho P.V.T., Lira R.N., Jark P.C., Melo J.F.P. \& Oliveira L.C.R. 2013. Apresentação atípica de tumor venéreo transmissível cutâneo em um cão. Vet. Zootec. 20(1):57-61.

Martinez M.A.R., Francisco G., Cabral L.S., Ruiz R.G. \& Festa Neto C. 2006. Genética molecular aplicada ao câncer cutâneo não melanoma. Anais Bras. Dermatol. 81(5):405-419. <http://dx.doi.org/10.1590/S036505962006000500003>

Meirelles A.E.W.B., Oliveira E.C., Rodrigues B.A., Costa G.R., Sonne L., Tesser E.S. \& Driemeier D. 2010. Prevalência de neoplasmas cutâneos em cães da região metropolitana de Porto Alegre/RS: 1.017 casos (2002-2007). Pesq. Vet. Bras. 30(11):968-973. <http://dx.doi.org/10.1590/S0100736X2010001100011>

Merlo D.F., Rossi L., Pellegrino C., Ceppi M., Cardellino U., Capurro C., Ratto A., Sambucco P.L., Sestito V., Tanara G. \& Bocchini V. 2008. Cancer incidence in pet dogs: findings of the animal tumor registry of Genoa, Italy. J. Vet. Intern Med. 22(4):976-984.<http://dx.doi.org/10.1111/j.1939-1676.2008.0133.x.> <PMid:18564221>

Modiano J.F. \& Breen M. 2007. The etiology of cancer, p.3-30. In: Withrow S.J. \& Vail D.M. (Eds), MacEwen's Small Animal Clinical Oncology. 4th ed. Saunders Elsevier, St Louis.

Mukaratirwa S., Chipunza J., Chitanga S., Chimonyo M. \& Bhebhe E. 2005. Canine cutaneous eoplasms: prevalence and influence of age, sex and site on the presence and potential malignancy of cutaneous neoplasms in dogs from Zimbabwe. J. S. Afr. Vet. Assoc. 76(2):59-62. <http://dx.doi. org/10.4102/jsava.v76i2.398><PMid:16108522>

Murphy S. 2006. Skin neoplasia in small animals. 1. Pinciples of diagnosis and management. Clin. Pract. 28(5):266-227.
Oliveria S.A., Saraiya M., Geller A.C., Heneghan M.K. \& Jorgensen C. 2006. Sun exposure and risk of melanoma. Arch. Dis. Childhood 91(2):131-138. <http://dx.doi.org/10.1136/adc.2005.086918> <PMid:16326797>

Pizzoni S., Sabattini S., Stefanello D., Dentini A., Ferrari R., Dacasto M., Giantin M., Laganga P., Amati M., Tortorella G. \& Marconato L. 2017. Features and prognostic imact of distant metastases in 45 dogs with de novo stage IV cutaneous mast cell tumours: a prospective study. Vet. Comp. Oncol. 16(1): 28-36. <PMid:28233400>

Priebe A.P.S., Riet-Correa G., Paredes L.J.A., Costa M.S.F., Silva C.D.C. \& Almeida M.B. 2011. Ocorrência de neoplasias em cães e gatos da mesorregião metropolitana de Belém, PA entre 2005 e 2010. Arq. Bras. Med. Vet. Zootec. 63(6):1583-1586. <http://dx.doi.org/10.1590/S0102-09352011000600042>

Scott D.W. \& Paradis M. 1990. A survey of canine and feline skin disorders seen in a university practice. Small Animal Clinic, University of Montréal, Saint-Hyacinthe, Québec (1987-1988). Can. Vet. J. 31(12):830-835. <PMid:17423707>

Sorenmo K.U., Kristiansen V.M., Cofone M.A., Shofer F.S., Breen A.-M., Langeland M., Mongil C.M., Grondahl A.M., Teige J. \& Goldschmidt M.H. 2009. Canine mammary gland tumors; a histological continuum from benign to malignant; clinical and histopathological evidence. Vet. Comp. Oncol. 7(3):162-172. <http://dx.doi.org/10.1111/j.1476-5829.2009.00184.x><PMid:19691645>

Souza T.M. 2005. Estudo retrospectivo de 761 tumores cutâneos em cães. Dissertação de Mestrado, Universidade Federal de Santa Maria, Santa Maria, RS. 280p.

Souza T.M., Fighera R.A., Irigoyen L.F. \& Barros C.S.L. 2006. Estudo retrospectivo de 761 tumores cutâneos em cães. Ciência Rural 36(2):555-560. <http:// dx.doi.org/10.1590/S0103-84782006000200030>

Torres de la Riva G., Hart B.L., Farver T.B., Oberbauer A.M., Messam L.L.M.V., Willits N. \& Hart L.A. 2013. Neutering dogs: Effects on joint disordes and cancers in Golden Retrievers. PLos One. 8(2):1-7. <http://dx.doi. org/10.1371/journal.pone.0055937><PMid:23418479>

Viana D.A., Pinto J.N., Souza L.P., Pacheco A.C.L., Morais G.B., Evangelista J.S.A.M. \& Silva L.D.M. 2014. Estudo retrospectivo das neoplasias mamárias caninas em Fortaleza e região metropolitana de 2003 a 2011. Ciênc. Anim. 24(1):35-45. 